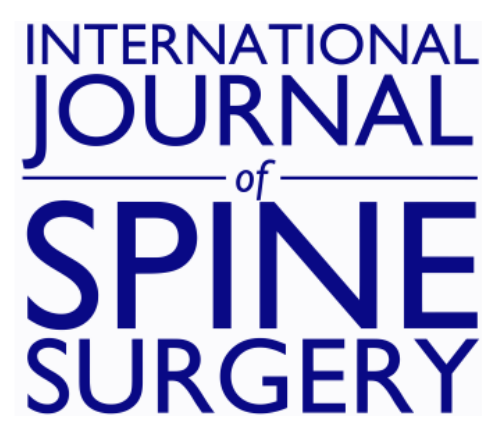

\title{
Biologics and Minimally Invasive Approach to TLIFs: What Is the Risk of Radiculitis?
}

ERIK WANG, CAROLYN STICKLEY, JORDAN MANNING, CHRISTOPHER G. VARLOTTA, DAINN WOO, ETHAN AYRES, EDEM ABOTSI, DENNIS VASQUEZ-MONTES, CHARLA R. FISCHER, JONATHAN STIEBER, MARTIN QUIRNO, THEMISTOCLES S. PROTOPSALTIS, PETER G. PASSIAS and AARON J. BUCKLAND

Int J Spine Surg 2020, 14 (5) 804-810

doi: https://doi.org/10.14444/7114

http://ijssurgery.com/content/14/5/804

This information is current as of April 26, 2023.

Email Alerts Receive free email-alerts when new articles cite this article. Sign up at: http://ijssurgery.com/alerts 


\title{
Biologics and Minimally Invasive Approach to TLIFs: What Is the Risk of Radiculitis?
}

\author{
ERIK WANG, BA, ${ }^{1}$ CAROLYN STICKLEY, BS, ${ }^{1}$ JORDAN MANNING, BA, ${ }^{1}$ CHRISTOPHER G. \\ VARLOTTA, BS, ${ }^{1}$ DAINN WOO, BS,${ }^{1}$ ETHAN AYRES, MPH,${ }^{1}$ EDEM ABOTSI, BA, ${ }^{1}$ DENNIS VASQUEZ- \\ MONTES, MS, ${ }^{1}$ CHARLA R. FISCHER, MD,${ }^{1}$ JONATHAN STIEBER, MD, ${ }^{1}$ MARTIN QUIRNO, MD,${ }^{1}$ \\ THEMISTOCLES S. PROTOPSALTIS, MD,${ }^{1}$ PETER G. PASSIAS, MD,${ }^{1}$ AARON J. BUCKLAND, MBBS, \\ FRACS $^{1,2}$ \\ ${ }^{1}$ Department of Orthopaedics, NYU Langone Orthopedic Hospital, New York, New York, ${ }^{2}$ Melbourne Orthopaedic Group, Melbourne, Australia
}

\begin{abstract}
Background: Bone morphogenetic protein (BMP) and allograft containing mesenchymal stem cells (live cell) are popular biologic substitutes for iliac crest autograft used in transforaminal lumbar interbody fusion (TLIF). Use of these agents in the pathogenesis of postoperative radiculitis remains controversial. Recent studies have independently linked minimally invasive (MIS) TLIF with increased radiculitis risk compared to open TLIF. The purpose of this study was to assess the rate of postoperative radiculitis in open and MIS TLIF patients along with its relationship to concurrent biologic adjuvant use.

Methods: Patients $\geq 18$ years undergoing single-level TLIF from June 2012 to December 2018 with minimum 1year follow-up were included. Outcome measures were rate of radiculitis, intra- and postoperative complications, revision surgery; length of stay (LOS), and estimated blood loss (EBL).

Results: There were 397 patients: 223 with open TLIFs, 174 with MIS TLIFs. One hundred and fifty-nine surgeries used bone morphogenetic protein (BMP), 26 live cell, 212 neither. Open TLIF: higher mean EBL, LOS, and Charlson Comorbidity Index (CCI) than MIS. Postoperative radiculitis in 37 patients (9.32\% overall): 16 cases MIS BMP (15.69\% of their cohort), 6 MIS without BMP (8.33\%), 5 open BMP $(8.77 \%), 10$ open without BMP (6.02\%). MIS TLIF versus open TLIF: no differences in 1-year reoperation rates, infection/wound complication, pseudarthrosis, or postoperative complication rate. BMP versus non-BMP: no differences in reoperation rates, infection/wound complication, pseudarthrosis, or postoperative complication rate. Multivariate logistic regression found that neither $\operatorname{BMP}(P=.109)$ nor MIS $(P=.314)$ was an independent predictor for postoperative radiculitis when controlled for age, gender, body mass index, and CCI. Using paired open and MIS groups ( $\mathrm{N}=168$ each) with propensity score matching, these variables were still not independently associated with radiculitis $(P=.174 \mathrm{BMP}, P=.398$ MIS). However, the combination of MIS with BMP was associated with increased radiculitis risk in both the entire patient cohort (odds ratio [OR]: 2.259 [1.117-4.569], $P=.023, \mathrm{~N}=397$ ) and PSM cohorts (OR: 2.196 [1.045-4.616], $P=.038, \mathrm{~N}=336$ ) compared to other combinations of surgical approach and biologic use.

Conclusion: Neither the MIS approach nor BMP use is an independent risk factor for post-TLIF radiculitis. However, risk of radiculitis significantly increases when they are used in tandem. This should be considered when selecting biological adjuvants for MIS TLIF.
\end{abstract}

Level of Evidence: 3

Biologics

Keywords: radiculitis, biologics, bone morphogenetic protein, minimally invasive surgery

\section{INTRODUCTION}

Recombinant human bone morphogenetic protein-2 (rhBMP-2) (Infuse, Medtronic Sofamor Danek, Memphis, Tennessee) and bone allograft containing human mesenchymal stem cells (live cell) (Osteocel Plus, NuVasive, San Diego, California) are popular biologic products used in spinal fusion procedures that have been used with increasing frequency. ${ }^{1-4}$ Both BMP and live cell are used as substitutes for autograft in order to reduce donor site pain with comparable fusion rates., ${ }^{3,5}$ Other proposed benefits are advocated in patients with poor-quality autogenous bone or those at higher risk for nonunion, such as elderly, osteoporotic patients, and those undergoing revision or multilevel surgeries. ${ }^{6}$ In particular, BMP has obtained US Food and Drug Administration approval for use in single-level anterior lateral interbody fusion (ALIF) 
inside an LT-CAGE. $^{3}$ However, BMP has been increasingly implemented in other lumbar spine procedures, particularly transforaminal lateral interbody fusion (TLIF) surgery, despite being offlabel for such usage. ${ }^{2}$ It is estimated that at least $85 \%$ of principal procedures using BMP in the United States are for off-label applications, of which approximately $30 \%$ are for primary TLIFs and an additional $1.6 \%$ are for revision TLIFs. ${ }^{2,3}$ In addition, approximately $30 \%$ of TLIFs in the United States use BMP. ${ }^{2}$

Despite its frequency, studies exploring clinical outcomes of BMP use in TLIFs are limited, particularly regarding the pathogenesis of postoperative radiculitis. The role that biologic adjuvants play in the development of radiculitis remains controversial. Several studies have reported on BMP-related complications in lumbar spine surgery, primarily heterotopic ossification, osteolysis, neurologic impairment, radiculitis, and formation of epidural cysts or seromas. Most of these complications have been theorized to result secondarily to an inflammatory response to BMP. ${ }^{7-12}$ In conjunction with these reports, other studies have correlated complication frequency and severity to use of higher BMP doses and attributed nerve root injury and radiculitis to application of BMP near neural structures. ${ }^{13-15}$ Nonetheless, most studies have been underpowered, and many have contradicted one another, resulting in varying recommendations regarding the use of BMP in TLIF surgeries. ${ }^{6}$

Recent studies have also independently linked minimally invasive (MIS) TLIFs with increased radiculitis risk compared to open TLIFs. ${ }^{5,16,17}$ In a 2013 study, Singh et $\mathrm{al}^{5}$ reported a $57 \%$ rate of transient postoperative radiculitis in 573 MIS TLIF patients, with radiculitis incidence independent of BMP dose, though they did not compare against a cohort of open TLIF patients. Epstein ${ }^{17}$ reviewed multiple lumbar surgical studies in a 2016 study and found a higher frequency of root injury and radiculitis in MIS operations compared to open techniques. There is, however, still a lack of conclusive data regarding postoperative radiculitis in relation to MIS versus open technique and use of biologics. Given the limitations of prior studies, our goal was to assess the rate of postoperative radiculitis in open and MIS TLIF patients along with its relationship to concurrent biologic adjuvant use.

\section{MATERIALS AND METHODS}

\section{Data Source and Inclusion Criteria}

A retrospective review from June 2012 to December 2018 was conducted for adult patients undergoing single-level TLIF at a single academic institution. Inclusion criteria were the following: adult patients ( $>18$ years) undergoing single-level TLIF at a single academic institution and a minimum 1-year follow-up.

\section{Surgical Technique}

For open TLIFs at our institution, a posterior midline incision is made over the lumbar spine, and paraspinal muscles are detached from the spinous processes, laminae, facet capsules, and transverse processes. Interbody placement occurs following a uni- or bilateral inferior facetectomy, unilateral superior facet resection, and discectomy. Fusion with pedicle screw placement occurs before or after TLIF placement, depending on surgeon preference. MIS TLIFs are performed via bilateral Wiltse approach, involving a paramedian incision $2-3 \mathrm{~cm}$ lateral to the midline, taken down to the lumbar dorsal fascia. This incision is made $2-2.5 \mathrm{~cm}$ in length longitudinally between the multifidus and longissimus muscles. Interbody preparation, along with bone grafting and cage placement, is performed using either serial dilators with tubular retractor placement or a pedicle screw-based retractor. When used, BMP was placed into the anterior contralateral disk space along with autograft and/or allograft prior to the insertion of the interbody cage to prevent BMP from leaking into the foramen. Live cell was placed in the same fashion. When expandable cages were used, these were backfilled with crushed cancellous autograft after expansion. BMP was not placed inside the TLIF cage. Complete facetectomy at the ipsilateral side and inferior facetectomy of the contralateral side is performed for fusion. Pedicle screws are placed percutaneously using guidewires and dilators either before or after TLIF placement, depending on surgeon preference.

\section{Data Collection and Outcome Measures}

Clinical data were gathered from each patient's electronic medical record and included operative notes, anesthesiology notes, and follow-up clinical notes. These were used to determine patient demographics, risk factors, preoperative diagnoses, operative dates and details, interbody graft prepa- 
ration and materials, perioperative outcomes, complications, any reoperations, and reasons for reoperation. Patients were assessed for development of radiculitis following their TLIF procedures. Postoperative radiculitis was defined as new radicular symptoms after initial improvement without evidence of residual neurological compression on postoperative imaging. Magnetic resonance imaging scans were obtained for patients who developed postoperative radicular symptoms to exclude compression, and computed tomography was ordered to ensure that there were no pedicle screw breaches. Demographic data collected included age, gender, body mass index (BMI) and Charlson Comorbidity Index (CCI). Surgical factors assessed included operative time, estimated blood loss (EBL), future reoperation within 1 year of index surgery, and reason for reoperation, open versus MIS technique to TLIF, and BMP or live cell use and dosage. Patients were also assessed for length of stay (LOS), presence of postoperative complications including infection, wound complications, revision surgery, and pseudarthrosis.

\section{Statistical Analysis}

Statistical analysis was performed using the Statistical Package for Social Sciences (version 23) (SPSS Inc, Chicago, Illinois). Univariate analysis and multivariate logistic regression analysis for categorical variables and 1-way analysis of variance for continuous variables were used to evaluate characteristics in association with development (or not) of postoperative radiculitis. Significance was set at $P<.05$. Propensity score matching (PSM) controlling for age, gender, BMI, and CCI was used to compare paired open and MIS groups.

\section{RESULTS}

\section{Patient Sample}

Three hundred and ninety-seven patients undergoing single-level TLIF at our institution from June 2012 to December 2018 were identified (52.90\% female and $47.10 \%$ male). Mean age was $59.33 \pm$ 13.49 years, BMI was $28.98 \pm 6.29 \mathrm{~kg} / \mathrm{m}^{2}$, CCI $2.29 \pm 1.92$. Two hundred and twenty-three patients underwent open TLIF, and 174 underwent MIS TLIF. One hundred and fifty-nine patients underwent TLIF with BMP, 26 underwent TLIF with live cell, and 212 underwent TLIF with neither biologic. Postoperative radiculitis occurred in 37 patients total $(9.32 \%$ overall): 16 cases of MIS TLIF with BMP (15.69\% of their cohort), 6 cases of MIS TLIF without BMP $(8.33 \%$ of their cohort), 5 cases of open TLIF with BMP $(8.77 \%$ of their cohort), and 10 cases of open TLIF without BMP $(6.02 \%$ of their cohort).

\section{Open TLIF Versus MIS TLIF}

Open TLIF patients were compared against MIS TLIF patients regardless of whether BMP was used. Open TLIF patients had a higher mean EBL (410.91 versus $212.28 \mathrm{~mL}, P<.001$ ), $\operatorname{LOS}$ (4.11 versus 3.20 days, $P<.001)$, and CCI (2.58 versus $1.93, P=$ .001) than MIS patients (Table 1). Of the 37 observed cases of postoperative radiculitis, 22 were MIS cases $(12.64 \%)$, and 15 were open cases $(6.73 \%, P=.046)$. There were no significant differences in 1-year reoperation rates $(9.42 \%$ versus $6.90 \%, P=.306)$, infection or wound complication (2.24\% versus $1.15 \%, P=.412)$, pseudarthrosis (2.69\% versus $4.02 \%, P=.464)$, or overall rate of postoperative complications $(30.94 \%$ versus $32.18 \%, P=.815)$ in open TLIF compared to MIS TLIF (Table 1).

\section{BMP Versus Non-BMP}

Of the 37 observed cases of postoperative radiculitis, 21 used BMP (13.21\%), and 16 did not use BMP $(6.72 \% ; P=.029)$. There were no significant differences in 1-year reoperation rates (10.08\% versus $5.66 \%, P=.118)$, infection or wound complication $(1.68 \%$ versus $1.89 \%, P=$ $.878)$, pseudarthrosis $(3.78 \%$ versus $2.52 \%, P=$ $.487)$, or overall rate of postoperative complications (31.09\% versus $32.08 \%, P=.836)$ between patients who received BMP and those who did not receive BMP (Table 1).

\section{Independence of Predictors of Postoperative Radiculitis}

Multivariate logistic regression was used to determine independent predictors for postoperative radiculitis. BMP use and open versus MIS technique were compared before and after matching for age, gender, BMI, and CCI. Neither BMP use nor MIS technique was found to be independent predictors of postoperative radiculitis (BMP $P=.109$, MIS $P=$ .314), but the combination of BMP and MIS technique predicted a risk for postoperative radiculitis (OR: 2.259[1.117-4.569], $P=.023, \mathrm{~N}=397$; 
Table 1. Comparison of demographics, perioperative outcomes, and postoperative complications by open versus minimally invasive (MIS) transforaminal lumbar interbody fusion (TLIF) and no bone morphogenetic protein (BMP) use versus BMP use.

\begin{tabular}{|c|c|c|c|c|c|c|c|}
\hline Parameter & Overall $(\mathrm{N}=397)$ & Open $(\mathrm{N}=223)$ & $\operatorname{MIS}(N=174)$ & $P$ Value & No $B M P(N=238)$ & $\mathrm{BMP}(\mathrm{N}=159)$ & $P$ Value \\
\hline \multicolumn{8}{|l|}{ Mean $\pm \mathrm{SD}$} \\
\hline Age & $59.33 \pm 13.49$ & $60.06 \pm 14.58$ & $58.39 \pm 11.92$ & 0.211 & $59.02 \pm 13.89$ & $59.79 \pm 12.88$ & .580 \\
\hline Body mass index & $28.98 \pm 6.29$ & $29.07 \pm 6.44$ & $28.86 \pm 6.11$ & 0.743 & $29.01 \pm 6.47$ & $28.94 \pm 6.05$ & .905 \\
\hline Charlson Comorbidity Index & $2.29 \pm 1.92$ & $2.58 \pm 1.96$ & $1.93 \pm 1.82$ & 0.001 & $2.34 \pm 2.05$ & $2.22 \pm 1.72$ & .529 \\
\hline Operative time, $\min$ & $230.28 \pm 82.59$ & $234.74 \pm 91.59$ & $224.56 \pm 69.21$ & 0.208 & $234.78 \pm 80.63$ & $223.53 \pm 85.25$ & .184 \\
\hline Estimated blood loss, $\mathrm{mL}$ & $324.04 \pm 300.41$ & $410.91 \pm 337.98$ & $212.28 \pm 193.79$ & $<0.001$ & $381.40 \pm 305.67$ & $237.63 \pm 271.14$ & $<.001$ \\
\hline Length of stay, d & $3.71 \pm 2.57$ & $4.11 \pm 2.52$ & $3.20 \pm 2.55$ & $<0.001$ & $3.93 \pm 2.67$ & $3.38 \pm 2.39$ & .035 \\
\hline \multicolumn{8}{|l|}{ Percentage } \\
\hline Gender (female) & 52.90 & 52.91 & 52.87 & 0.993 & 47.80 & 56.30 & .096 \\
\hline Radiculitis & $9.32(\mathrm{n}=37)$ & $6.73(\mathrm{n}=15)$ & $12.64(\mathrm{n}=22)$ & 0.046 & $6.72(\mathrm{n}=16)$ & $13.21(\mathrm{n}=21)$ & .029 \\
\hline Future reoperation & $8.31(\mathrm{n}=33)$ & $9.42(\mathrm{n}=21)$ & $6.90(\mathrm{n}=12)$ & 0.306 & $10.08(\mathrm{n}=24)$ & $5.66(\mathrm{n}=9)$ & .118 \\
\hline Infection/wound complication & $1.76(\mathrm{n}=7)$ & $2.24(\mathrm{n}=5)$ & $1.15(\mathrm{n}=2)$ & 0.412 & $1.68(\mathrm{n}=4)$ & $1.89(\mathrm{n}=3)$ & .878 \\
\hline Pseudarthrosis & $3.27(\mathrm{n}=13)$ & $2.69(\mathrm{n}=6)$ & $4.02(\mathrm{n}=7)$ & 0.464 & $3.78(\mathrm{n}=9)$ & $2.52(\mathrm{n}=4)$ & .487 \\
\hline Postoperative complication & $31.49(\mathrm{n}=125)$ & $30.94(n=69)$ & $32.18(\mathrm{n}=56)$ & 0.815 & $31.09(\mathrm{n}=74)$ & $32.08(\mathrm{n}=51)$ & .836 \\
\hline
\end{tabular}

Table 2). After propensity matching open and MIS groups $(\mathrm{N}=168$ each), these variables were still not independently associated with radiculitis (BMP $P=$ .174 , MIS $P=.398)$. However, the combination of MIS with BMP was associated with increased radiculitis risk after matching (OR: 2.196[1.0454.616], $P=.038, \mathrm{~N}=336$; Table 3) compared to other combinations of surgical approach and biologic use.

\section{DISCUSSION}

The use of BMP in lumbar spine surgery has increased ever since the US Food and Drug Administration approved its use in single-level ALIFs. This has particularly been the case for TLIF surgeries despite the off-label indication of BMP. Potential benefits of using BMP in TLIF surgeries include reduced donor graft site pain and morbidity and equal or better fusion rates achieved comparison to iliac crest bone graft or cancellous allograft. ${ }^{3,5,6}$ A meta-analysis exploring graft material in MIS TLIFs conducted by Parajón et al ${ }^{18}$ reported a higher fusion rate of $96.6 \%$ in patients receiving BMP compared to $92.5 \%$ in a pooled group of patients receiving any combination of grafts excluding BMP. Although BMP has proven beneficial in TLIFs, it comes with unique disadvan-

Table 2. Comparison of combinations of approach and biologic use and their odds of association with postoperative radiculitis among all patients $(\mathrm{N}=397)$.

\begin{tabular}{lccc}
\hline Approach and Biologic Used & OR & $\mathbf{9 5 \%}$ CI & $\boldsymbol{P}$ Value \\
\hline MIS with BMP & 2.259 & $1.117-4.569$ & .023 \\
MIS without BMP & 0.795 & $0.314-2.014$ & .628 \\
Open with BMP & 1.045 & $0.380-2.871$ & .993 \\
Open without BMP & 0.511 & $0.239-1.097$ & .085
\end{tabular}

Abbreviations: BMP, bone morphogenetic protein; CI, confidence interval; MIS, minimally invasive; OR, odds ratio. tages, such as the potential development of heterotopic ossification, osteolysis, neural defects, and radiculitis, which have been attributed to inflammatory mechanisms. ${ }^{3,6}$ MIS lumbar spine surgeries have also been linked to increased incidence of radiculitis and other complications in comparison to open techniques. ${ }^{16,17}$ Nonetheless, findings in various studies have been conflicting. Because of this, we sought to assess the rate of postoperative radiculitis in open and MIS TLIF surgeries in conjunction with its relationship to use of biologic adjuvants. To our knowledge, this is the first study to compare BMP, live cell, and nonbiologic use along with open versus MIS techniques concurrently in patients undergoing TLIF surgery.

We found an overall postoperative radiculitis rate of $9.32 \%$, occurring in 37 out of 397 patients. Comparing biologic use independent of open or MIS technique, 21 radiculitis cases occurred in BMP patients (13.21\% of all BMP patients), and 16 cases occurred in non-BMP patients $(6.72 \%$ of non-BMP patients). None of the patients who received live cell during surgery developed radiculitis, though the number of patients included was limited. These rates are similar to prior reports. Rihn et al, ${ }^{3}$ Mummaneni et $\mathrm{al}^{19}{ }^{19}$ and $\mathrm{Khan}$ et $\mathrm{al}^{20}$ all previously compared postoperative radiculitis rates between

Table 3. Comparison of combinations of approach and biologic use and their odds of association with postoperative radiculitis among propensity score matched patients $(\mathrm{N}=336)$

\begin{tabular}{lccc}
\hline Approach and Biologic Used & OR & $\mathbf{9 5 \%}$ CI & $\boldsymbol{P}$ Value \\
\hline MIS with BMP & 2.196 & $1.045-4.616$ & .038 \\
MIS without BMP & 0.689 & $0.252-1.885$ & .468 \\
Open with BMP & 0.844 & $0.243-2.939$ & .791 \\
Open without BMP & 0.601 & $0.267-1.354$ & .219 \\
\hline
\end{tabular}

Abbreviations: BMP, bone morphogenetic protein; CI, confidence interval; MIS, minimally invasive; OR, odds ratio. 
BMP $(14 \%, 9 \%$, and $8.4 \%$, respectively) and nonBMP groups $(3 \%, 5.3 \%$, and $2 \%$, respectively), though there were no statistical differences. Comparing open versus MIS TLIFs independent of biologic use, 22 of these cases occurred in MIS patients $(12.64 \%$ of all MIS patients), while 15 occurred in open patients $(6.73 \%$ of all open patients). Looking at MIS versus open technique in conjunction with biologic adjuvant status, there were 16 cases of radiculitis in patients who underwent MIS TLIF with BMP, which is $15.69 \%$ of their cohort. In addition, there were 6 radiculitis cases among those who underwent MIS TLIF without BMP $(8.33 \%$ of their cohort), 5 cases for open TLIF with BMP $(8.77 \%$ of their cohort $)$, and 10 cases for open TLIF without BMP $(6.02 \%$ of their cohort).

In comparing the aforementioned variables as risk factors for developing postoperative radiculitis, we first compared open TLIFs against MIS TLIFs independent of whether BMP was used. Unsurprisingly, open TLIF patients had a higher mean EBL and longer average LOS compared to MIS patients. Mean CCI was also higher in open TLIF patients, reflecting that MIS TLIF patients tended to be healthier than their open TLIF counterparts. There were no significant differences in 1-year reoperation rates, infection or wound complication, pseudarthrosis, or overall rate of postoperative complications between open and MIS patients. We also compared risk factors for patients who underwent TLIF with BMP against those who received no BMP. Because live cell was not involved in any radiculitis cases and was used much less frequently, we compared BMP use against non-BMP use rather than BMP versus live cell versus neither. BMP patients had significantly lower mean EBL and LOS than non-BMP patients, but this was likely attributable to overlap of MIS patients in the BMP cohort. We found no significant differences in age, BMI, CCI, operative time, reoperation rates, infection or wound complication, pseudarthrosis, or overall rate of postoperative complications between BMP and non-BMP patients. This lack of differences in rates of short-term complications is similar to previous findings.

To determine independent predictors for postoperative radiculitis, we used multivariate logistic regression controlling for age, gender, BMI, and CCI. In this analysis, we found that neither BMP $(P$ $=.109)$ nor MIS $(P=.314)$ was an individually independent predictor for postoperative radiculitis. When open and MIS groups were propensity score matched for age, gender, BMI, and CCI, MIS technique and BMP use were still not independently associated with radiculitis. This differs from recent findings that have linked BMP use in adult spinal deformity surgeries with increased odds of developing radiculitis or other neurological complications, though those reports included multiple types of surgery in addition to TLIFs. ${ }^{7}$ However, we analyzed groupings of MIS or open technique with biologic use, comparing patient groups of MIS with BMP, MIS without BMP, open with BMP, and open without BMP, and found that the combination of MIS with BMP was associated with increased radiculitis risk compared to other combinations. This was the case for both the entire patient cohort of 397 patients and the PSM group of 336 total patients. There are several reasons why this combination of MIS technique and BMP use may lead to radiculitis. First, our study may be underpowered to detect a difference for MIS and BMP as independent risk factors; however, this is a large cohort of patients. There is limited exposure in the MIS technique, and unlike the open TLIF technique, interlaminar distraction is not performed. This may result in more nerve root traction or irritation during disc space preparation and cage insertion. When added to the inflammatory response to BMP, the authors believe that this added insult results in higher incidence of radiculitis.

Prior studies have suggested methods for reducing neurologic complications when using BMP in TLIF surgery. Lykissas et $\mathrm{al}^{21}$ described local 10-mg dexamethasone administration in addition to soaking the adjunct on the carrier sponge for at least 45 minutes prior to insertion for optimized BMP retention. Crandall et $\mathrm{al}^{22}$ described placing BMP as far anterior and contralateral to the TLIF cage as possible, then backfilling local autograft around the cage to minimize BMP access to the spinal canal. Although the mechanism leading to radiculitis in TLIF patients remains poorly understood, better informing patients about surgical risks and taking additional steps to prevent symptoms may reduce morbidity and improve outcomes.

Our study has some limitations. Because this was a retrospective cohort study, it has all of the accompanying limitations of such a study. In particular, our ability to track diagnoses and resolving of radiculitis depended on reading indi- 
vidual clinic notes, which may have been incomplete or introduced selection or information bias. Additionally, characterizing severity and duration of postoperative radiculitis may not have been satisfactorily accomplished from clinical follow-up notes alone. We also had a small sample size, as evidenced by the large confidence intervals, and a follow-up of 1 year may have limited patient inclusion. Future studies could benefit from having a larger sample size and also creating a standardized protocol for evaluating postoperative radicular symptoms and tracking them over time. Nonetheless, our study is, to our knowledge, the largest retrospective cohort study comparing postoperative effects of BMP and non-BMP TLIF surgeries while also being the first to study BMP use in tandem with open versus MIS technique as it relates to postoperative radiculitis. The rates of radiculitis in our patients with varying exposure to MIS technique and BMP in their TLIF surgery were similar to previous studies. While neither BMP use nor MIS approach showed independent significance in increasing radiculitis risk, their combination did. This should be considered when selecting biological adjuvants for MIS TLIF surgeries. Patients undergoing MIS TLIF with BMP should be informed of higher risks of radiculitis as part of their surgical consent.

\section{CONCLUSIONS}

The overall postoperative radiculitis rate is $9.32 \%$ for single-level TLIF patients at our institution, and rates are higher for patients who underwent MIS TLIF, received BMP in their procedure, or both. Neither MIS approach nor BMP use are independent risk factors for post-TLIF radiculitis, but the risk of radiculitis significantly increases when they are used in tandem. This should be considered when selecting biological adjuvants for MIS TLIF.

\section{REFERENCES}

1. Skovrlj B, Marquez-Lara A, Guzman JZ, Qureshi SA. A review of the current published spinal literature regarding bone morphogenetic protein-2: an insight into potential bias. Curr Rev Musculoskelet Med. 2014;7(3):182-188. doi:10.1007/s12178-0149221-3.

2. Ong KL, Villarraga ML, Lau E, Carreon LY, Kurtz SM, Glassman SD. Off-label use of bone morphogenetic proteins in the United States using administrative data. Spine (Phila Pa 1976). 2010;35(19):1794-1800. doi:10.1097/BRS.0b013e3181ecf $6 \mathrm{e} 4$.

3. Khan TR, Pearce KR, McAnany SJ, Peters CM, Gupta MC, Zebala LP. Comparison of transforaminal lumbar interbody fusion outcomes in patients receiving rhBMP-2 versus autograft. Spine J. 2018;18(3):439-446. doi:10.1016/j. spinee.2017.08.230.

4. McAnany SJ, Ahn J, Elboghdady IM, et al. Mesenchymal stem cell allograft as a fusion adjunct in one- and two-level anterior cervical discectomy and fusion: a matched cohort analysis. Spine J. 2016;16(2):163-167. doi:10.1016/j.spinee.2015. 02.037 .

5. Singh K, Nandyala S V, Marquez-Lara A, et al. Clinical sequelae after rhBMP-2 use in a minimally invasive transforaminal lumbar interbody fusion. Spine J. 2013;13(9):11181125. doi:10.1016/j.spinee.2013.07.028.

6. Villavicencio AT, Burneikiene S. RhBMP-2-induced radiculitis in patients undergoing transforaminal lumbar interbody fusion: relationship to dose. Spine J. 2016;16(10):1208-1213. doi:10.1016/j.spinee.2016.06.007.

7. Poorman GW, Jalai CM, Boniello A, Worley N, McClelland S, Passias PG. Bone morphogenetic protein in adult spinal deformity surgery: a meta-analysis. Eur Spine $J$. 2017;26(8):2094-2102. doi:10.1007/s00586-016-4841-5.

8. Resnick DK, Choudhri TF, Dailey AT, et al. Guidelines for the performance of fusion procedures for degenerative disease of the lumbar spine. Part 16: bone graft extenders and substitutes. J Neurosurg Spine. 2005;2(6):733-736. doi:10.3171/ spi.2005.2.6.0733.

9. US Food and Drug Administration. InFuse Bone Graft LT-Cage: Summary of Safety and Effectiveness. Washington, DC: US Food and Drug Administration; 2002. http://www.ac cessdata.fda.gov/cdrh_docs/pdf/P000058b.pdf.

10. McClellan JW, Mulconrey DS, Forbes RJ, Fullmer N. Vertebral bone resorption after transforaminal lumbar interbody fusion with bone morphogenetic protein (rhBMP-2). $J$ Spinal Disord Tech. 2006;19(7):483-486. doi:10.1097/01.bsd. 0000211231.83716.4b.

11. Kang DG, Hsu WK, Lehman RA. Complications associated with bone morphogenetic protein in the lumbar spine. Orthopedics. 2016;40(2):e229-e237. doi:10.3928/0147744720161213-06.

12. Chrastil J, Patel AA. Complications associated with posterior and transforaminal lumbar interbody fusion. $\mathrm{J} \mathrm{Am}$ Acad Orthop Surg. 2012;20(5):283-291. doi:10.5435/JAAOS-2005-283.

13. Crandall DG, Revella J, Patterson J, Huish E, Chang M, McLemore R. Transforaminal lumbar interbody fusion with rhBMP-2 in spinal deformity, spondylolisthesis, and degenerative disease - part 1: large series diagnosis related outcomes and complications with 2- to 9-year follow-up. Spine (Phila $\mathrm{Pa}$ 1976). 2013;38(13):1128-1136. doi:10.1097/BRS.0b013e318288 $64 \mathrm{e} 6$.

14. Wong DA, Kumar A, Jatana S, Ghiselli G, Wong K. Neurologic impairment from ectopic bone in the lumbar canal: a potential complication of off-label PLIF/TLIF use of bone morphogenetic protein-2 (BMP-2). Spine J. 2008;8(6):10111018. doi:10.1016/j.spinee.2007.06.014.

15. Dmitriev AE, Lehman RA, Symes AJ. Bone morphogenetic protein-2 and spinal arthrodesis: the basic science perspective on protein interaction with the nervous system. Spine J. 2011;11(6):500-505. doi:10.1016/j.spinee.2011.05.002.

16. Epstein N. More nerve root injuries occur with minimally invasive lumbar surgery: Let's tell someone. Surg Neurol Int. 2016;7(4):96. doi:10.4103/2152-7806.174896. 
17. Epstein N. More nerve root injuries occur with minimally invasive lumbar surgery, especially extreme lateral interbody fusion: a review. Surg Neurol Int. 2016;7(4):83. doi:10.4103/2152-7806.174895.

18. Parajón A, Alimi M, Navarro-Ramirez R, et al. Minimally invasive transforaminal lumbar interbody fusion: meta-analysis of the fusion rates. What is the optimal graft material? Clin Neurosurg. 2017;81(6):958-971. doi:10.1093/neu ros/nyx 141 .

19. Mummaneni PV, Pan J, Haid RW, Rodts GE. Contribution of recombinant human bone morphogenetic protein-2 to the rapid creation of interbody fusion when used in transforaminal lumbar interbody fusion: a preliminary report. J Neurosurg Spine. 2009; 2004;1(1):19-23. doi:10.3171/ spi.2004.1.1.0019.

20. Rihn JA, Patel R, Makda J, et al. Complications associated with single-level transforaminal lumbar interbody fusion. Spine J. 2009;9(8):623-629. doi:10.1016/j.spinee.2009. 04.004 .

21. Lykissas MG, Aichmair A, Sama AA, Hughes AP, Lebl DR, Cammisa FP, et al. Nerve injury and recovery after lateral lumbar interbody fusion with and without bone morphogenetic protein-2 augmentation: a cohort-controlled study. Spine J. 2014;14(2):217-224. doi:10.1016/j.spinee.2013.06.109.

22. Crandall DG, Revella J, Patterson J, Huish E, Chang M, McLemore R. Transforaminal lumbar interbody fusion with
rhBMP-2 in spinal deformity, spondylolisthesis, and degenerative disease - part 2: BMP dosage-related complications and long-term outcomes in 509 patients. Spine (Phila Pa 1976). 2013;38(13):1137-1145. doi:10.1097/BRS.0b013e3182880298.

Disclosures and COl: The authors report no conflicts of interest. Each institution obtained approval from their local institutional review board to enroll patients in this retrospective study.

Corresponding Author: Aaron J. Buckland, MBBS, FRACS, Spine Research Center, Department of Orthopaedic Surgery, NYU Langone Health, 306 East 15th Street, Ground Floor, New York, NY 10003. Phone: (646) 794-8643; Fax: (929) 455-9241; Email: aaronbuckland@me.com.

Published 11 November 2020

This manuscript is generously published free of charge by ISASS, the International Society for the Advancement of Spine Surgery. Copyright (C) 2020 ISASS. To see more or order reprints or permissions, see http://ijssurgery.com. 